\title{
Bases para una ley de pastizales de regiones áridas y semiáridas de la Argentina
}

\author{
Marcos H. Easdale \\ Instituto de Investigaciones Forestales y Agropecuarias Bariloche (IFAB, INTA-CONICET), Río Negro, Argentina.
}

\begin{abstract}
Resumen. Los pastizales proveen múltiples servicios ecosistémicos de alto valor para la sociedad, tanto local como globalmente, pero se encuentran amenazados por procesos de degradación, el impacto del cambio climático y modificaciones en el uso del suelo, que afectan su conservación. A pesar de ello, la preservación de los pastizales ha recibido escasa atención por parte de los organismos gubernamentales y no gubernamentales en la Argentina. Este artículo tiene por objetivo proponer algunos conceptos y principios asociados a los ecosistemas de pastizal de regiones áridas y semiáridas, tomando como ejemplo el contexto patagónico, como bases para discutir el diseño de una ley de presupuestos mínimos de protección ambiental de pastizales en dichas regiones de la Argentina. Para ello, se revisan brevemente algunos antecedentes respecto al abordaje de la desertificación, la caracterización de pastizales y los territorios pastoriles en la Patagonia. Desde una perspectiva integral, se sugiere que la elaboración de una herramienta de política pública como la que se propone requiere de un enfoque biocultural de la conservación de los territorios pastoriles. En este sentido, algunos principios que rigen este enfoque son utilizados para definir criterios de zonificación. A modo de conclusión, se resaltan algunas premisas generales para buscar acuerdos entre objetivos de preservación de la biodiversidad y la promoción de la diversidad cultural, que caracterizan este tipo de regiones.
\end{abstract}

Palabras clave: conservación, desertificación, biocultural, territorios pastoriles, sistemas socioecológicos

\begin{abstract}
Aвstract. Basis for a law of rangelands from arid and semiarid regions of Argentina. Rangelands provide multiple ecosystem services of high value to society, both locally and globally, but are threatened by degradation processes, the impact of climate change and land-use change, affecting their conservation. Despite this, the preservation of rangelands has received little attention from governmental and non-governmental organizations in Argentina. This article aims to propose some concepts and principles associated with rangelands from arid and semiarid regions, using as an example the Patagonian context, as preliminary bases to discuss the design of a law of minimum budgets for the environmental protection of rangelands in these regions of Argentina. To do this, some background information on the approach to desertification, characterization of rangelands and pastoral territories in Patagonia are briefly reviewed. From a comprehensive perspective, it is suggested that the development of a public policy tool such as the one proposed requires a biocultural approach to the conservation of pastoral territories. In this sense, some principles that govern this approach are used to define zoning criteria. As a conclusion, some general premises are highlighted to seek agreements between the objectives of preserving biodiversity and promoting cultural diversity, which characterize these kind of regions.
\end{abstract}

Keywords: conservation, desertification, biocultural, grassland territories, social-ecological systems

\section{INTRODUCCIÓN}

La protección de los pastizales de regiones áridas y semiáridas ha recibido escasa atención por parte de los organismos gubernamentales y no gubernamentales en la Argentina, a excepción de algunas iniciativas locales. A diferencia de los bosques nativos, la falta de acuerdos más generales para la elaboración de herramientas de política pública que se enfoquen en la conservación de pastizales, se evidencia en la ausencia de una ley de presupuestos mínimos para su protección. Por ello, se considera pertinente iniciar un debate en la materia, con la finalidad de avanzar en el desarrollo de una propuesta de ley.
Los pastizales fueron definidos y caracterizados de muy diversas maneras, lo cual denota que no se cuenta con una definición universal (Lund 2007). En este artículo, se consideran ecosistemas de pastizal a aquellas tierras no cultivadas, cuya vegetación está dominada por gramíneas, arbustos, hierbas o graminoides, que son pastoreadas por ganado doméstico o animales silvestres (Stoddart et al. 1975; Holecheck et al. 2004). El término pastizal incluye las praderas de pastos altos y pastos cortos, matorrales, sabanas, desiertos, estepas y tundras, que comprenden entre 30 y $40 \%$ de la superficie terrestre libre de hielo de la Tierra (dependiendo de la definición y la fuente de datos, Asner et al. 2004; Lund 
2007). Esta propuesta focaliza en los pastizales ubicados en regiones áridas y semiáridas $(0.05<$ Índice de Aridez<0.5) (Reynolds et al. 2007), que representan el 65\% de la superficie global de las tierras secas (Safriel and Adeel 2005), y ocupan una proporción significativa del territorio de la Argentina. Su relevancia radica en que son fuente de múltiples servicios ecosistémicos de alto valor para la sociedad, tanto local como globalmente (Anderson et al. 2011). En particular, proveen de forraje para el ganado, fuente de calor en viviendas (Cardoso et al. 2012), flora ornamental y usos medicinales (Ladio and Lozada 2009). Por otro lado, regulan el funcionamiento de cuencas hídricas y procesos de erosión de suelo (Mazzoni and Rabassa 2013), proveyendo de hábitat a multiplicidad de especies, muchas de ellas endémicas (Cei 1969; Andrade and Monjeau 2014; López et al. 2018). Debido a la gran extensión geográfica, regiones como las estepas patagónicas constituyen vastos territorios cubiertos por vegetación que reservan carbono (Nosetto et al. 2006), y controlan funciones clave como el ciclado de nutrientes y la infiltración (Aguiar and Sala 1999). A su vez, constituyen la base del principal medio de vida y desarrollo cultural e identitario de numerosas comunidades rurales (Pastur et al. 2016; Easdale and Aguiar 2018). Finalmente, son ambientes que prestan un servicio recreativo cada vez más valorado por habitantes que desarrollan un estilo de vida urbano y menos conectado con la naturaleza.

Los pastizales ocupan la mayor proporción de la superficie en la Patagonia (Argentina), región que es utilizada como ejemplo en esta propuesta. En dicha región, los pastizales están afectados, en distintos grados, por procesos históricos de desertificación (del Valle et al. 1998), siendo creciente tanto la amenaza del cambio climático como el desarrollo de actividades económicas que conllevan cambios en el uso de la tierra (Mazzoni and Vázquez 2009). Desde un punto de vista socio-productivo, la mayor proporción de los pastizales se encuentra bajo uso ganadero ovino, caprino o bovino (Villagra et al. 2015). Mientras que intensidades intermedias de pastoreo demostraron tener diversos beneficios para los ambientes de pastizal (Oñatibia et al. 2015), estudios locales evidenciaron que el sobrepastoreo es uno de los factores relevantes en promover procesos de degradación (Bisigato and Bertiller 1997; Perelman et al. 1997; Oñatibia and Aguiar 2016; Enriquez and Cremona 2018). Dicha producción ganadera se desarrolla de manera heterogénea en el espacio, junto con fauna nativa y exótica, la cual también depende de los pastizales como sustento. Es por ello que la competencia por alimento entre ovinos y guanacos (Baldi et al. 2001; Pedrana et al. 2010; Marino et al. 2020) o las interacciones entre ganadería y carnívoros (Gaspero et al. 2018; Llanos et al. 2020) están entre los conflictos principales. Por otro lado, la implementación de otros usos de la tierra (e.g., minera, forestal) constituyen alternativas de uso más recientes, pero también con alto impacto social y ambiental. En particular, la actividad hidrocarburífera ha incrementado los niveles de degradación ambiental en determinadas zonas, con crecimiento acelerado en los últimos 50 años (Mazzonia and Vázquez 2009). Finalmente, los sistemas extensivos de base pastoril están asociados, en muchas zonas, a comunidades rurales cuya identidad cultural se referencia con los pastizales. Por esta razón, constituyen una base de soberanía alimentaria y ocupación territorial hacia el interior de vastos territorios de la Patagonia. Este artículo tiene por objetivo proponer algunos conceptos y principios asociados a los pastizales en el contexto patagónico, que sirvan como bases preliminares para discutir el diseño de una ley de presupuestos mínimos de protección ambiental de pastizales de regiones áridas y semiáridas en la Argentina.

\section{Algunos antecedentes: UnA MIRADA PATAGÓNICA}

\section{La desertificación: Un problema complejo que requiere un abordaje socioecológico}

Uno de los principales consensos académicos y políticos de la últimas dos décadas fue el reconocimiento de que la desertificación es un problema complejo que se debe abordar desde una perspectiva que integre dinámicas sociales y ecológicas (Reynolds and Stafford Smith 2002). Esto significa que las regiones áridas y semiáridas como la Patagonia, en tanto sistemas socioecológicos, requieren abordajes rigurosos de la complejidad multidimensional, la interdependencia, los cambios no lineales y a través de escalas espaciales y temporales (Reynolds et al. 2007).

A pesar de este progreso indudable y prometedor, la transición desde perspectivas disciplinarias hacia el surgimiento de enfoques más integrales es un complejo proceso social en sí mismo y requiere de tiempo (Clark and Dickson 2003). En este contexto, la 
desertificación no es una excepción, aspecto evidenciado en que diferentes desarrollos teóricos y metodológicos para su estudio están todavía en debate (Thomas 1997; Verón et al. 2006; Vogt et al. 2011). En la Patagonia, las principales contribuciones aplicadas refieren a aspectos biofísicos de la evaluación de la degradación (e.g., Verón and Paruelo 2010; Gaitán et al. 2018), herramientas para el seguimiento de la desertificación (e.g., Easdale et al. 2019; Oliva et al. 2019), o sus aspectos socioproductivos (Andrade 2012). Sin embargo, los estudios socioeconómicos aún tienen escasos vínculos con los análisis biofísicos (Torres et al. 2015). Como un paso haciaunaintegraciónen suabordaje, se propuso un marco para combatir la desertificación denominado 'Paradigma de Desarrollo de las Tierras Secas' (Reynolds et al. 2007), orientado a comprender el funcionamiento de los sistemas socioecológicos en regiones áridas y semiáridas. Se propuso que estas regiones se caracterizan por un conjunto único de características que deben tenerse en cuenta para estructurar el análisis de los cambios y para desarrollar un sistema de seguimiento y evaluación integral (Reynolds et al. 2011). En particular, se identificaron siete características como relacionadas causalmente en el desarrollo de un síndrome del desierto, sustentadas en un determinismo biofísico. El síndrome consta de tres características descritas como causales: 1) variabilidad climática, 2) recursos escasos, y 3) escasa población, que influyen en otras cuatro características: 4) lejanía, 5) variabilidad social, 6) prevalencia de conocimiento local, y 7) diferencias culturales. (Stafford Smith 2008). Luego, una perspectiva coevolutiva sobre el papel de impulsores políticos, sociales y económicos mundiales, y sus impactos locales en regiones pastoriles áridas y semiáridas, complementó dicha perspectiva sobre las causas y las consecuencias. En particular, se propuso que: a) la aplicación mundial de paradigmas occidentales en el manejo de pastizales, b) la Revolución Verde basada en combustibles fósiles, y c) la implementación de instituciones capitalistas utilizadas para regular el comercio agrícola y las herramientas y políticas asociadas, enfatizaron el síndrome de los desiertos (Easdale and Domptail 2014).

Estos marcos concurrentes demuestran los esfuerzos recientes para conceptualizar de manera más integral el problema de la desertificación. De todas formas, ante el proceso de degradación de pastizales se suele poner de relieve una tensión o crisis entre preservar la biodiversidad de los pastizales y promover la sociodiversidad en términos culturales, organizativos y económicos (Aagesen 2000; Golluscio et al. 2010). De hecho, iniciativas recientes como la degradación neutral de la tierra impulsada por Naciones Unidas continúan enfocando las soluciones en evitar la degradación o revertir procesos actuales en términos meramente biofísicos. El foco de acción prioriza la implementación de prácticas de manejo sustentable de tierras (Grainger 2015). Sin embargo, otras opciones vinculadas a promover el desarrollo social y territorial de las comunidades rurales que gestionan dichos ecosistemas, como vías para obtener el mismo fin, aparecen con mucho menos énfasis (Easdale 2016).

\section{Las ecorregiones y los pastizales en la Patagonia}

Existe una larga tradición de estudios que caracterizaron los ecosistemas patagónicos tanto regional como localmente, y que sirven como base para diseñar una zonificación y caracterizarla en términos biofísicos. Los primeros esfuerzos regionales refieren a aproximaciones florísticas para clasificar el paisaje, reconociendo que las características de la vegetación son típicamente los aspectos más relevantes que definen a los ecosistemas, dando como resultado clasificaciones que se presentan en mapas fitogeográficos (Soriano 1956; Cabrera 1971). Posteriormente, algunas propuestas avanzaron con mapas fisonómicoflorísticos de la vegetación espontánea, con el objetivo de distinguir las unidades de vegetación dentro de las provincias fitogeográficas (Movia et al. 1982; Roig et al. 1985; León et al. 1998, actualizado por Oyarzabal et al. [2018]). Otras propuestas discriminaron biozonas, tomando como base de clasificación las características funcionales de la vegetación, principalmente asociadas a variaciones temporales de la productividad primaria neta aérea (Soriano and Paruelo 1992; Paruelo et al. 1998). Con un abordaje holístico, otras propuestas caracterizaron regiones ecológicas integrando características fisonómico-florísticas de la vegetación con aspectos geomorfológicos, de suelo o clima (Soriano 1983; Bran 1992, actualizado por Bran et al. [2005]), o incluyendo también un conjunto de variables climáticas y en la manifestación de procesos naturales y de degradación de origen antrópico (del Valle et al. 1995).

En una cobertura de mayor detalle, estudios locales o zonales caracterizaron la relación 
entre la vegetación y factores ambientales en estepas (e.g., Bertiller et al. 1995; Golluscio et al. 1982) y en humedales (e.g., Gaitán et al. 2011; Irisarri et al. 2012). Este tipo de ambientes, denominados localmente 'mallines', con características azonales y baja representación espacial en términos de superficie, constituyen sitios estratégicos de conservación (Crego et al. 2014) y para el manejo pastoril (Golluscio et al. 1998). Por último, algunas propuestas incorporaron, junto con la información de la vegetación, datos de presencia de pequeños mamíferos como variables clasificatorias del paisaje (Monjeau et al. 1998). Estos estudios reflejan la necesidad de integrar distintas extensiones espaciales y resoluciones de los datos, ambos aspectos que deben ser tenidos en cuenta durante la realización de zonificaciones orientadas a discriminar categorías de importancia en la conservación. Por ejemplo, tomar como base el esfuerzo reciente en la identificación de áreas prioritarias e irremplazables, en términos de la distribución de especies y ecosistemas en la Patagonia (Chehébar et al. 2013).

\section{Una aproximación a los territorios pastoriles de la Patagonia}

Una perspectiva complementaria al de las ecorregiones, definidas en términos fundamentalmente biofísicos, es la de identificar y caracterizar los territorios pastoriles de la Patagonia, para el cual hay menos antecedentes. En el contexto de esta propuesta, se entiende por territorio a la unión o vínculo de un sentido (o significado) con un lugar determinado, o sea, al espacio apropiado y valorizado, tanto simbólica como instrumentalmente, por grupos humanos (Raffestin 1980). Entonces, el territorio pastoril es el sistema socioecológico que reúne o integra una comunidad de base pastoril con el medio natural que habita. Desde esta perspectiva se podrían diferenciar territorios pastoriles o regiones socioecológicas a lo largo de toda la región árida y semiárida de la Patagonia (Easdale and Aguiar 2012), al igual que en otras regiones del país (Vallejos et al. 2019). En este sentido, las caracterizaciones basadas en las Zonas Agroeconómicas Homogéneas de la Patagonia (Schorr and Segui 2008; Easdale and Madariaga 2009), constituyen un antecedente en esta dirección. De todas maneras, dichos trabajosincluyeronla producción agropecuaria e información poblacional en su conjunto, y no se enfocaron en el uso pastoril.
Algunos ejemplos zonales permiten referenciar la noción de territorio pastoril. Por caso, en el centro-norte de la provincia de Neuquén predomina la actividad denominada trashumancia, que aglomera a familias crianceras, algunas pertenecientes a comunidades indígenas. La trashumancia se basa en movimientos estacionales entre sitios de pastoreo preestablecidos y con condiciones ambientales contrastantes, que involucran zonas áridas y montañosas. Durante el invierno e inicio de la primavera, las familias y sus animales permanecen en zonas bajas denominadas invernadas. A fines de primavera, se trasladan por huellas y caminos de arreo de uso común, hacia pastizales de altura ubicados en zonas cordilleranas, denominadas veranadas (Bendini et al. 2004). El sistema trashumante involucra los pastizales de invernada y veranada y los caminos de arreo que los interconectan, conformando una red regional de movimiento y uso del paisaje (Easdale et al. 2016). Dicho ciclo anual de movimiento ocurre de manera sincrónica entre fases ecológicas y sociales (Pérez León et al. 2020). Esto significa que cualquier propuesta de protección y uso sustentable de pastizales en dicho territorio debe considerar esta conectividad socioecológica.

Por otro lado, el sur de la provincia de Neuquén, la denominada Línea Sur de Río Negro y el noroeste de Chubut presentan una matriz mixta de productores capitalizados y estancias de amplias superficies, con predominancia de sistemas ganaderos extensivos ovinos-bovinos, y familias ganaderas de pequeña escala y comunidades indígenas, con sistemas mixtos ovinos-caprinos-bovinos (Easdale et al. 2009; Golluscio et al. 2010; Villagra et al 2015). En general, el sistema de pastoreo involucra distintos ambientes de pastizales, que son utilizados con pastoreo continuo o con una lógica móvil, pero en el marco de límites espaciales definidos bajo un uso predial. Por último, el centro-sureste de Chubut, Santa Cruz y Tierra del Fuego presentan una dominancia de sistemas ovinos extensivos, con predominancia de productores capitalizados y estancias (Andrade et al. 2010), que presentan mayor proporción de límites definidos y titularidad de tierras. En estos casos, el manejo pastoril también involucra distintos ambientes, bajo un manejo productivo muy extensivo. La identificación y caracterización de los territorios pastoriles en la Patagonia (y 
otras regiones del país) podría constituir un objetivo de una propuesta de ley, en tanto línea de base, el cual requiere de procesos participativos que involucren al sector público y privado. Este objetivo debe alcanzar consensos sobre los espacios construidos y en disputa, refrendando los procesos históricos, culturales e identitarios asociados al uso pastoril.

\section{BASES PARA UNA PROPUESTA DE LEY}

\section{Protección del patrimonio biocultural de territorios pastoriles}

Los pastizales de la Patagonia se ubican entre los ecosistemas con menor proporción de superficie bajo regímenes de protección, en particular de Parques Nacionales (Martin and Chehébar 2001). Desde un abordaje socioecológico de la problemática de la desertificación y de los territorios pastoriles, la protección de los pastizales requiere de una perspectiva biocultural. La motivación para avanzar en un régimen de presupuestos mínimos de protección no puede estar únicamente sustentada en la protección de la biodiversidad local, sino también en la participación activa de las comunidades pastoriles, cuyas prácticas culturales han coevolucionado con estos ecosistemas, en una ventana de tiempo humana (Kallis and Norgaard 2010; Easdale and Domptail 2014). El emergente de esta coevolución está reflejado en las configuraciones y funcionamientos socioecológicos, adaptados a las condiciones particulares de un territorio. Norgaard (1981) propone que "los sistemas sociales y ambientales coevolucionan de manera que los sistemas ambientales reflejan las características de los sistemas sociales: sus conocimientos, valores, organizaciones sociales y tecnologías; mientras que los sistemas sociales reflejan las características de los sistemas ambientales: su mezcla de sociedades, tasas de productividad, variación espacial y temporal, y su resiliencia". Dado que los sistemas socioecológicos no cambian de una manera predecible, lineal, ni de un modo aditivo, comprender el funcionamiento de sus componentes no significa que se pueda predecir su comportamiento general (Walker and Salt 2006).

La perspectiva biocultural tiene como principio un enfoque cultural de la conservación, desde un abordaje holístico. La naturaleza constituye un componente indisociable de la cultura, y los aspectos éticos que constituyen las interrelaciones entre el hábitat y los habitantes, configuran la identidad y el sustento de vida. Por ello, la conservación biocultural busca el bienestar social y ecológico, a través de la conservación de la diversidad biológica y cultural, y sus interrelaciones (Rozzi 2013). En este contexto, existe un reconocimiento creciente a las prácticas culturales realizadas por comunidades pastoriles (generalmente desestimadas), asociadas no sólo al uso ganadero, sino también a una perspectiva multifuncional del paisaje y de las comunidades vegetales, la conservación de lugares religiosos o especies consideradas sagradas. Por caso, la trashumancia ha sido recientemente declarada Patrimonio Cultural Inmaterial de la Humanidad (UNESCO 2019). La noción de patrimonio busca reconocer las prácticas culturales vinculadas a la modalidad de pastoreo consistente en desplazamientos estacionales por rutas de arreo, entre ecosistemas distantes y disímiles. Este tipo de reconocimiento es un paso adelante para avanzar con otro tipo de prácticas culturales asociadas al vínculo entre la actividad pastoril y los ecosistemas de pastizal. Sin embargo, es importante destacar que, para quienes poseen $\mathrm{y}$ reproducen socialmente el patrimonio, la cultura es dinámica y heterogénea, fuera del alcance de medidas mercantiles, dado que se refiere a las formas en las cuales la gente da sentido a su vida y a los valores que la sustentan. En cambio, quienes administran la cultura suelen diseñar políticas destinadas a masificar, homogeneizar y mercantilizar lo cultural en tanto capital, por ejemplo, a través del reconocimiento y la gestión patrimonial (Andrade Orellana et al. 2020). Esta propuesta considera prioritaria una perspectiva biocultural dinámica, ejercida por los propios sujetos que desarrollan una actividad pastoril en un territorio, revisando sus adaptaciones o cambios emergentes (Easdale and Aguiar 2018). No debiera circunscribirse a un conjunto determinado de prácticas pastoriles y culturales, listadas en un modelo estanco y homogéneo de manejo de los pastizales. A su vez, debieran evitarse las propuestas unidireccionales, en tanto recetas, originadas en sectores científico-técnicos, así como también las reivindicaciones indiscutibles de prácticas sostenidas por las comunidades locales involucradas.

El patrimonio biocultural requiere de un reconocimiento de la diversidad, en un proceso de coconstrucción e integración de conocimientos en cada lugar (Raymond et 
al. 2010). Esta perspectiva es convergente con la propuesta de reconocer la diversidad de valoraciones respecto a la contribución que hace la naturaleza a las personas (Díaz et al. 2018), cuyo abordaje podría servir de base para avanzar en las distintas dimensiones bioculturales del pastoralismo. La participación de las comunidades pastoriles debe ser activa, tanto en la mediación de prácticas y propuestas tecnológicas como en la gestión de sus territorios, en plataformas de innovación donde también convergen el sector científico-técnico y decisores y ejecutores de política pública (Morriss et al. 2006). Por ejemplo, las Unidades Ejecutoras Provinciales (UEP) de la Ley Ovina (25422) y la Ley Caprina (26141/06) están conformadas con la participación de representantes de productores y de instituciones públicas. Asimismo, en el marco de la Ley 3016 (2016) de trashumancia en la provincia de Neuquén, se generaron comisiones de arreo zonales, conformadas por representantes de los crianceros trashumantes e instituciones provinciales y nacionales vinculadas al sector. Estas comisiones tienen el objetivo de priorizar acciones de restauración y desarrollo de infraestructura, orientadas a mejorar las condiciones generales de las huellas y rutas de arreo, preservar el ambiente y garantizar el tránsito de las familias y su ganado. Tomando estos antecedentes, en cada territorio pastoril, las unidades ejecutoras del patrimonio biocultural podrían referenciarse en comisiones de gestión participativa.

\section{Principios para la protección biocultural de territorios pastoriles}

Los principios que podrían regir la necesidad de presupuestos mínimos de protección biocultural de territorios pastoriles, tomando el ejemplo de la Patagonia, se incluyen a continuación.

Principio ético. Los pastizales tienen valor intrínseco y se debe propender al mantenimiento de la salud de los ecosistemas y los procesos ecológicos involucrados. Las especies involucradas deben existir y persistir, en tanto principio fundamental de la conservación biológica (Soulé 1985; Van Dyke 2008).

Principio ecológico. Los pastizales han coevolucionado con herbívoros y su dinámica ecológica necesita de la interacción pastizalherbívoros como proceso para preservar estructuras y funciones ecosistémicas para futuras generaciones (Adler et al. 2005;
Hernández et al. 2019), bajo criterios de uso sustentable de los pastizales y de la fauna.

Principio de mitigación del cambio climático. Dada su extensión territorial, los pastizales de la Patagonia son ecosistemas irremplazables, con capacidad de captura y reservorio de carbono y nutrientes que impactan globalmente, y regulan procesos hídricos y eólicos potencialmente erosivos regional y localmente, bajo criterios de manejo sustentable (Gaitán et al. 2014; Peri et al. 2018).

Principio de producción agropecuaria. La ganadería extensiva de base pastoril es la principal actividad productiva, de la cual dependen economías locales por ingresos económicos, abastecimiento de alimentos y fuentes de trabajo (Andrade 2012; Villagra et al. 2015). En muchas zonas, la actividad pastoril preserva recursos genéticos locales (Lanari et al. 2008).

Principio de identidad cultural y soberanía alimentaria. Los pastizales constituyen la base del principal medio de vida y de reproducción de aspectos culturales e identitarios de comunidades rurales en distintos territorios de la Patagonia. En este sentido, algunas características distintivas de los sujetos sociales pastoriles son la soberanía alimentaria y atributos agroecológicos, identidad pastoril (apego a los animales), diversidad cultural (e.g., crianceros, comunidades indígenas, estancieros), movilidad social y productiva (trashumancia, dinámica rural-urbana) y ocupación territorial (Golluscio et al. 2010; Bendini and Steimbreger 2011; Pastur et al. 2016; Easdale and Aguiar 2018).

\section{Marco normativo}

El marco normativo de la presente propuesta lo constituye la Ley Nacional № 25675, llamada Ley General de Ambiente de la República Argentina, la cual se creó en el año 2002 con el fin principal de brindar presupuestos mínimos para la gestión del ambiente. La ley está enmarcada en el artículo 41 de la Constitución Nacional, que declara que el ambiente "debe ser apto para el desarrollo humano y para que las actividades productivas satisfagan las necesidades presentes sin comprometer a las generaciones futuras". Este marco normativo sentó las bases para el desarrollo de las siguientes leyes nacionales vigentes: a) Ley 26331 de presupuestos mínimos de protección ambiental de los bosques nativos, 
b) Ley 26639 de presupuestos mínimos para la preservación de los glaciares y del ambiente periglacial, y c) Ley 27520 de presupuesto mínimos ambientales para la adaptación y mitigación del cambio climático global.

A su vez, al momento de redactar esta propuesta se han comenzado a discutir nuevamente algunos proyectos para impulsar una ley de presupuestos mínimos de protección ambiental y uso racional y sostenible de humedales, luego de haber perdido estado parlamentario a fines del año 2015. Es evidente que una propuesta focalizada en los ambientes de humedales, y las leyes vigentes mencionadas, tienen muchos elementos en común con un proyecto orientado a proteger y promover la preservación de pastizales, que deberán ser tratados particularmente. Por ejemplo, algunos humedales como los mallines en la Patagonia, constituyen ecosistemas de pastizal, los cuales deberían quedar contenidos en una propuesta como la presente.

Una propuesta de zonificación de los pastizales

Con la intención de avanzar en una propuesta, he tomado las categorías de zonificación acordadas en la Ley 26331 de bosques nativos, adaptándolas al contexto de los territorios pastoriles de la Patagonia y a la perspectiva de protección del patrimonio biocultural. En particular, propongo las siguientes categorías, las cuales combinan criterios de valor ambiental de conservación, tipo de tenencia y gestión de la tierra (Figura 1).

Categoría I (rojo, Conservación Ambiental Estricta de áreas irremplazables). Sectores de muy alto valor de conservación que no deben tener intervenciones que eliminen o sustituyan la vegetación natural y que deben mantenerse

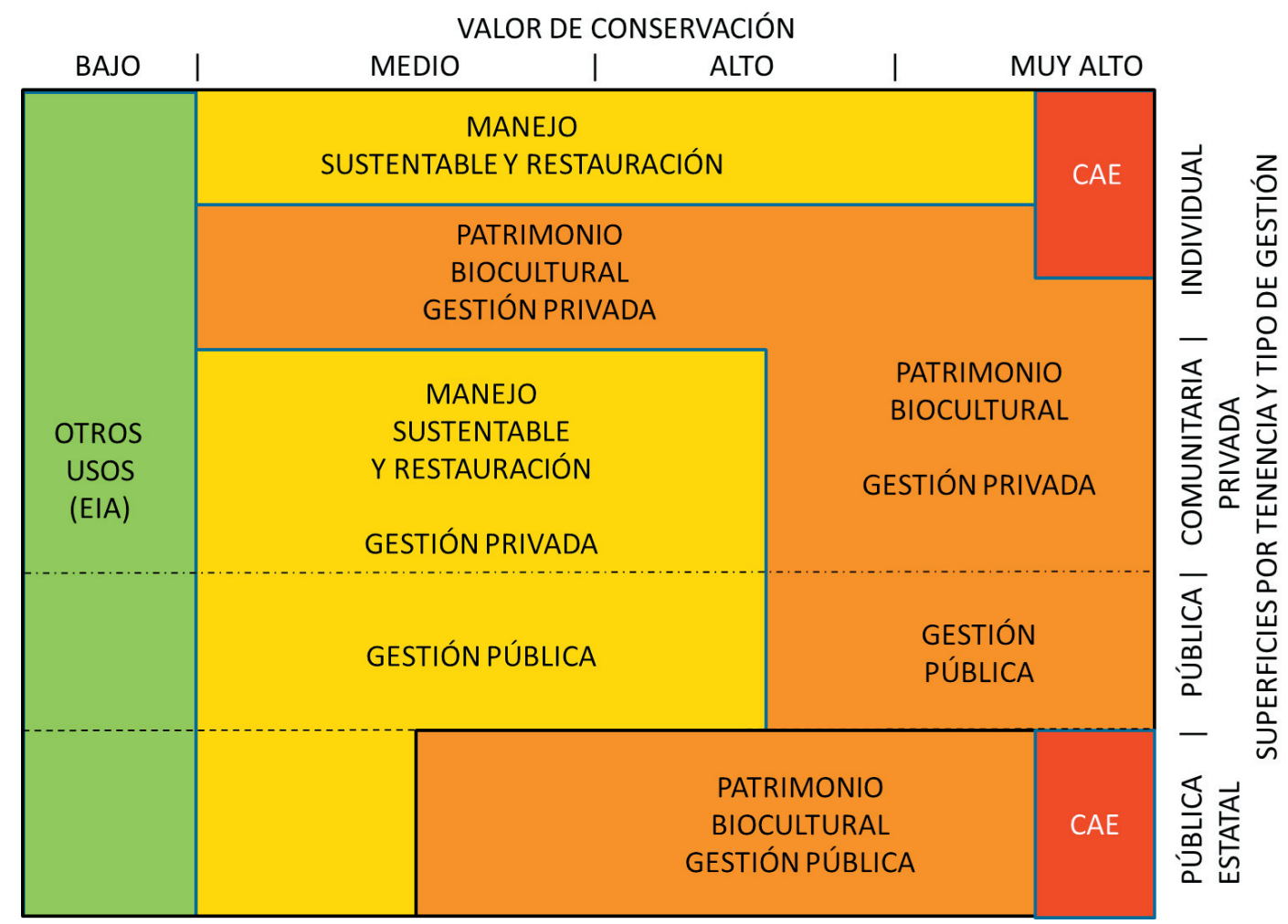

Figura 1. Categorías de zonificación de pastizales en relación con el valor de conservación y con la superficie bajo tenencia estatal o privada (comunitaria o individual) - línea cortada -, y gestión pública o privada - línea cortada y punto-. Referencias: Conservación Ambiental Estricta (CAE) y Evaluación de Impacto Ambiental (EIA). Categoría I (Conservación Ambiental Estricta, rojo), II (Patrimonio Biocultural y Manejo Sustentable, naranja), III (Manejo Sustentable y Restauración, amarillo), IV (Otro usos, verde).

Figure 1. Zoning categories of pastoral ecosystems in relation to the conservation value and the surface under State or private tenure (community or individual) - dashed line - , and public or private management - dashed+period line - . References: Strict Environmental Conservation (CAE) and Environmental Impact Assessment (EIA). Category I (Strict Environmental Conservation, red), II (Biocultural Heritage and Sustainable Management, orange), III (Sustainable Management and Restoration, yellow), IV (Other uses, green). 
como pastizales para siempre, y para uso de fauna nativa. Incluirá las reservas naturales y sus áreas circundantes, y áreas no protegidas que tengan valores biológicos sobresalientes, o sitios que protejan cuencas hídricas de importancia (nacientes de ríos y arroyos).

Categoría II (naranja, Patrimonio Biocultural y Manejo Sustentable de áreas prioritarias). Sectores de alto o medio valor de conservación, que pueden estar degradados pero que si se los restaura pueden tener un valor alto de conservación, cuya gestión está bajo custodio ambiental y cultural de comunidades rurales basados en agricultura familiar, indígenas o colectivos sociales con referencia identitaria pastoril. Estas áreas no pueden tener intervenciones que eliminen o sustituyan la vegetación natural ni la base cultural, y aplicará la propuesta de patrimonio biocultural. Podrán ser sometidas a los siguientes usos: aprovechamiento sostenible, turismo, recolección e investigación científica, con participación ineludible de la comunidad local involucrada.

Categoría III (amarillo, Manejo Sustentable y Restauración de áreas prioritarias). Sectores de alto o medio valor de conservación, que pueden estar degradados pero que si se los restaura pueden tener un valor alto de conservación. Estas áreas no pueden tener intervenciones que eliminen o sustituyan la vegetación natural, pero podrán ser sometidas a los siguientes usos: aprovechamiento sostenible, turismo, recolección e investigación científica.

Categoría IV (verde, Otros usos). Sectores de bajo valor de conservación que pueden transformarse parcialmente o en su totalidad, con la previa realización de una Evaluación de Impacto Ambiental.

\section{Conclusiones}

Los pastizales proveen múltiples servicios ecosistémicos en gran parte del territorio argentino, pero constituyen ecosistemas que se encuentran amenazados por diversos factores que afectan su conservación. Por ello, se considera que es necesario iniciar una discusión respecto al diseño de una ley de presupuestos mínimos de protección ambiental de pastizales de regiones áridas y semiáridas de la Argentina. Se propone avanzar en un enfoque biocultural de la conservación y una gestión participativa de los territorios pastoriles basada en la integración de conocimientos, desde un abordaje socioecológico de los problemas y las soluciones. Para tal fin, es necesario reconocer la diversidad de valoraciones respecto a la contribución de los ecosistemas de pastizal en la vida de las personas y los contextos culturales asociados. Tomando como ejemplo el contexto de la Patagonia, se sugiere la combinación de algunos criterios sociales y ecológicos, que servirían como base para elaborar una propuesta integral de zonificación, la cual podría ser utilizada en otras regiones del país (e.g., Monte, Puna). Una propuesta de ley debería enfatizar la búsqueda de consensos entre objetivos de preservación de la biodiversidad y la promoción de la diversidad cultural, que caracterizan las regiones áridas y semiáridas con predominio de pastizales.

Agradecimientos. Agradezco a dos revisores anónimos y a los editores asociados por sus contribuciones y sugerencias, que permitieron mejorar significativamente el mensaje del artículo. Agradezco también los comentarios de Dardo R. López y Martín R. Aguiar, vertidos en una primera versión del artículo.

\section{REFERENCIAS}

Aagesen, D. 2000. Crisis and conservation at the end of the world: sheep ranching in Argentine Patagonia. Environmental Conservation 27:208-215. https://doi.org/10.1017/S0376892900000229.

Adler, P. B., D. G. Milchunas, O. E. Sala, I. C. Burke, and W. K. Lauenroth. 2005. Plant traits and ecosystem grazing effects: comparison of US sagebrush steppe and Patagonian steppe. Ecological Applications 15(2):774-792. https: //doi.org/10.1890/04-0231.

Aguiar, M. R., and O. E. Sala. 1999. Patch structure, dynamics and implications for the functioning of arid ecosystems. Trends in Ecology and Evolution 14(7):273-277. https://doi.org/10.1016/S0169-5347(99)01612-2.

Anderson, D. L., G. L. Bonvissuto, M. A. Brizuela, G. Chiossone, A. F. Cibils, S. M. Cid, I. Feldman, R. C. F. Grecco, C. Kunst, M. Oesterheld, G. E. Oliva, J. M. Paruelo, H. R. Peinetti, and E. S. Villagra. 2011. Perspectives on Rangeland Management Education and Research in Argentina. Rangelands 33(1):2-12. https://doi.org/10.2111/RANGELANDSD-10-00016.1.

Andrade, L., V. Bedacarratx, and R. Álvarez. 2010. Producción ovina extensiva en la Patagonia Austral: el caso de la zona centro de Santa Cruz. Mundo Agrario 11:21.

Andrade, L. 2012. Producción y ambiente en la Meseta Central de Santa Cruz, Patagonia austral en Argentina: 
desencadenantes e impacto de la desertificación. Ambiente y Desarrollo 16(30):73-92.

Andrade, A., and A. Monjeau. 2014. Patterns in community assemblage and species richness of small mammals across an altitudinal gradient in semi-arid Patagonia, Argentina. Journal of Arid Environments 106:18-26. https://doi.org/ 10.1016/j.jaridenv.2014.02.004.

Andrade Orellana, S., S. Cárate Tandalla, and S. Freire García. 2020. Patrimonio Cultural Inmaterial: apropiación y resistencias. Pontificia Universidad Católica del Ecuador. Pp. 112.

Asner, G. P., A. J. Elmore, L. P. Olander, R. E. Martin, and A. T. Harris. 2004. Grazing systems, ecosystem responses, and global change. Annual Review of Environment and Resources 29:261-299. https://doi.org/10.1146/annurev.ene rgy.29.062403.102142.

Baldi, R., S. Albon, and D. Elston. 2001. Guanacos and sheep: evidence for continuing competition in arid Patagonia. Oecologia 129(4):561-570. https://doi.org/10.1007/s004420100770.

Bendini, M., P. Tsakoumagkos, and C. Nogués. 2004. Los crianceros trashumantes del Neuquén. Pp. 23-39 en M Bendini and C Alemany. Crianceros y chacareros en la Patagonia. Cuaderno GESA 5. Buenos Aires, La Colmena.

Bendini, M. I., and N. G. Steimbreger. 2011. Persistencia campesina en el norte de la Patagonia: Movilidades espaciales y cambios en la organización social del trabajo. Cuadernos de Desarrollo Rural 8(66):125-151.

Bertiller, M. B., N. O. Elissalde, C. M. Rostagno, and G. E. Defossé. 1995. Environmental patterns and plant distribution along aprecipitation gradient in western Patagonia. Journal of Arid Environments 29(1):85-97. https://doi.org/10.1016/ S0140-1963(95)80066-2.

Bisigato, A. J., and M. B. Bertiller. 1997. Grazing effects on patchy dryland vegetation in northern Patagonia. Journal of Arid Environments 36:639-653. https://doi.org/10.1006/jare.1996.0247.

Bran, D. 1992. Las regiones ecológicas de la Patagonia y sus principales formaciones vegetales. Comunicación Técnica INTA 3:1-12.

Bran, D., G. Oliva, P. Rial, J. Escobar, C. López, F. Umaña, J. Ayesa, and N. Elissalde. 2005. Regiones Ecológicas Homogéneas de la Patagonia Argentina. Comunicación Técnica Relevamiento Integrado $N^{\circ} 132$, Área Recursos Naturales. INTA. Pp. 12.

Cabrera, A. L. 1971. Fitogeografía de la República Argentina. Boletín de la Sociedad Argentina de Botánica 14:1-42.

Cardoso, M. B., A. H. Ladio, and M. Lozada. 2012. The use of firewood in a Mapuche community in a semi-arid region of Patagonia, Argentina. Biomass and Bioenergy 46:155-164. https://doi.org/10.1016/j.biombioe.2012.09.008.

Cei, J. M. 1969. The Patagonian telmatobiid fauna of the volcanic Somuncura Plateau of Argentina. Journal of Herpetology 3:1-18. https://doi.org/10.2307/1563219.

Chehébar, C., A. Novaro, G. Iglesias, S. Walker, M. Funes, M. Tammone, and K. Didier. 2013. Identificación de áreas de importancia para la biodiversidad en la estepa y el monte de Patagonia. ErreGé y Asociados imprenta. Pp.112.

Clark, W. C., and N. M. Dickson. 2003. Sustainability science: the emerging research program. Proceedings of the National Academy of Sciences 100:8059-8061. https://doi.org/10.1073/pnas.1231333100.

Crego, R. D., C. K. Nielsen, and K. A. Didier. 2014. Climate change and conservation implications for wet meadows in dry Patagonia. Environmental Conservation 41(2):122-131. https://doi.org/10.1017/S037689291300026X.

del Valle, H. F., J. C. Labraga, and J. Goergen. 1995. Biozonas de la Región Patagónica. En Evaluación del Estado Actual de la Desertificación en Areas Representativas de la Patagonia: Informe Final de la etapa I. Pp. 37-55. INTA-GTZ, Río Gallegos-Trelew-Puerto Madryn-Bariloche.

del Valle, H. F., N. O. Elissalde, D. A. Gagliardini, and J. Milovich. 1998. Status of desertification in the Patagonian region: Assessment and mapping from satellite imagery. Arid Land Research and Management 12(2):95-121. https: //doi.org/10.1080/15324989809381502.

Díaz, S., U. Pascual, M. Stenseke, B. Martín-López, R. T. Watson, Z. Molnár, R. Hill, K. M. Chan, I. A. Baste, K. A. Brauman, S. Polasky, A. Church, M. Lonsdale, A. Larigauderie, P. W. Leadley, A. P. E. van Oudenhoven, F. van der Plaat, M. Schröter, S. Lavorel, Y. Aumeeruddy-Thomas, E. Bukvareva, K. Davies, S. Demissew, G. Erpul, P. Failler, C. A. Guerra, Ch. L. Hewitt, H. Keune, S. Lindley, and Y. Shirayama. 2018. Assessing nature's contributions to people. Science 359(6373):270-272. https://doi.org/10.1126/science.aap8826.

Easdale, M. H., M. Aguiar, M. Román, and S. Villagra. 2009. Comparación socioeconómica de dos regiones biofísicas: los sistemas ganaderos de la provincia de Río Negro, Argentina. Cuadernos de Desarrollo Rural 6(62):26-26.

Easdale, M. H., and M. C. Madariaga. 2009. Zonas Agroeconómicas Homogéneas de Patagonia Norte: Neuquén y Río Negro. Serie Estudios Socioeconómicos de la Sustentabilidad de los Sistemas de Producción y Recursos Naturales, INTA. Pp. 111.

Easdale, M. H., and M. R. Aguiar. 2012. Regional forage production assessment in arid and semi-arid rangelandsA step towards social-ecological analysis. Journal of Arid Environments 83:35-44. https://doi.org/10.1016/ j.jaridenv.2012.03.002.

Easdale, M. H., and S. E. Domptail. 2014. Fate can be changed! Arid rangelands in a globalizing world-A complementary co-evolutionary perspective on the current 'desert syndrome'. Journal of Arid Environments 100:52-62. https://doi.org/ 10.1016/j.jaridenv.2013.10.009.

Easdale, M. H. 2016. Zero net livelihood degradation-the quest for a multidimensional protocol to combat desertification. SOIL 2(2):129-134. https://doi.org/10.5194/soil-2-129-2016.

Easdale, M. H., M. R. Aguiar, and P. Paz. 2016. A social-ecological network analysis of Argentinean Andes transhumant pastoralism. Regional Environmental Change 16(8):2243-2252. https://doi.org/10.1007/s10113-015-0917-8.

Easdale, M. H., and M. R. Aguiar. 2018. From traditional knowledge to novel adaptations of transhumant pastoralists 
the in face of new challenges in North Patagonia. Journal of Rural Studies 63:65-73. https://doi.org/10.1016/ j.jrurstud.2018.09.001.

Easdale, M. H., C. Fariña, S. Hara, N. P. León, F. Umaña, P. Tittonell, and O. Bruzzone. 2019. Trend-cycles of vegetation dynamics as a tool for land degradation assessment and monitoring. Ecological Indicators 107:105545. https://doi.org/ 10.1016/j.ecolind.2019.105545.

Enriquez, A. S., and M. V. Cremona. 2018. Particulate organic carbon is a sensitive indicator of soil degradation related to overgrazing in Patagonian wet and mesic meadows. Wetlands Ecology and Management 26(3):345-357. https: //doi.org/10.1007/s11273-017-9577-4.

Gaitán, J. J., C. R. López, and D. E. Bran. 2011. Vegetation composition and its relationship with the environment in mallines of north Patagonia, Argentina. Wetlands Ecology and Management 19(2):121-130.

Gaitán, J. J., G. E. Oliva, D. E. Bran, F. T. Maestre, M. R. Aguiar, E. G. Jobbágy, G. G. Buono, D. Ferrante, V. B. Nakamatsu, G. Ciari, and J. M. Salomone. 2014. Vegetation structure is as important as climate for explaining ecosystem function across Patagonian rangelands. Journal of Ecology 102(6):1419-1428. https://doi.org/10.1111/1365-2745.12273.

Gaitán, J. J., D. E. Bran, G. E. Oliva, M. R. Aguiar, G. G. Buono, D. Ferrante, V. Nakamatsu, G. Ciari, J. M. Salomone, V. Massara, and G. G. Martínez. 2018. Aridity and overgrazing have convergent effects on ecosystem structure and functioning in Patagonian rangelands. Land Degradation and Development 29(2):210-218. https://doi.org/10.1002/ ldr.2694.

Gáspero, P. G., M. H. Easdale, J. A. Pereira, V. Fernández-Arhex, and J. Von Thüngen. 2018. Human-carnivore interaction in a context of socio-productive crisis: Assessing smallholder strategies for reducing predation in North-west Patagonia, Argentina. Journal of Arid Environments 150:92-98. https://doi.org/10.1016/j.jaridenv.2017.12.005.

Golluscio, R. A., R. J. C. León, and S. B. Perelman. 1982. Caracterización fitosociológica de la estepa del Oeste de Chubut. Su relación con el gradiente ambiental. Boletín de la Sociedad Argentina de Botánica 21:299-324.

Golluscio, R. A., V. A. Deregibus, and J. M. Paruelo. 1998. Sustainability and range management in the Patagonian steppes. Ecología Austral 8(02):265-284.

Golluscio, R. A., M. E. Román, A. Cesa, D. Rodano, H. Bottaro, M. I. Nieto, A. Betelú, and L. A. Golluscio. 2010. Aboriginal settlements of arid Patagonia: Preserving bio-or sociodiversity? The case of the Mapuche pastoral Cushamen Reserve. Journal of Arid Environments 74(10):1329-1339. https://doi.org/10.1016/j.jaridenv.2010.05.012.

Grainger, A. 2015. Is land degradation neutrality feasible in dry areas? Journal of Arid Environments 112:14-24. https: //doi.org/10.1016/j.jaridenv.2014.05.014.

Hernández, F., C. Ríos, and H. L. Perotto-Baldivieso. 2019. Evolutionary history of herbivory in the Patagonian steppe: The role of climate, ancient megafauna, and guanaco. Quaternary Science Reviews 220:279-290. https://doi.org/10.1016/ j.quascirev.2019.07.014.

Holechek, J. R., D. Pieper, and C. H. Herbel. 2004. Range management: principles and practices. 5th ed. Prentice Hall. Upper Saddle River, NJ, EUA. Pp. 587.

Kallis, G., and R. B. Norgaard. 2010. Coevolutionary ecological economics. Ecological Economics 69(4):690-699. https: //doi.org/10.1016/j.ecolecon.2009.09.017.

Ladio, A. H., and M. Lozada. 2009. Human ecology, ethnobotany and traditional practices in rural populations inhabiting the Monte region: resilience and ecological knowledge. Journal of Arid Environments 73(2):222-227. https://doi.org/10.1016/j.jaridenv.2008.02.006.

Lanari, M. R., E. Domingo, and L. Gallo. 2008. Caracterización genética de la cabra criolla neuquina. Archivos de Zootecnia 57(219):365-368.

León, R. J. C, D. Bran, M. Collantes, J. M. Paruelo, and A. Soriano. 1998. Grandes unidades de vegetación de la Patagonia extra andina. Ecología Austral 8:123-141.

Ley 3016. Provincia de Neuquén, Argentina. 2016. URL: tinyurl.com/2v3cbrws (último acceso: 15 de mayo de 2021).

Llanos, R., A. Andrade, and A. Travaini. 2020. Puma and livestock in central Patagonia (Argentina): from ranchers' perceptions to predator management. Human Dimensions of Wildlife 25(1):1-16. https://doi.org/10.1080/ 10871209.2019.1668987.

Lund, H. G. 2007. Accounting for the world's rangelands. Rangelands 29:3-10. https://doi.org/10.2111/1551501X(2007)29[3:AFTWR]2.0.CO;2.

Marino, A., V. Rodríguez, and N. M. Schroeder. 2020. Wild guanacos as scapegoat for continued overgrazing by livestock across southern Patagonia. Journal of Applied Ecology 57(12):2393-2398. https://doi.org/10.1111/1365-2664.13536.

Martin, C. E., and C. Chehébar. 2001. The national parks of Argentinian Patagonia - Management policies for conservation, public use, rural settlements, and indigenous communities. Journal of the Royal Society of New Zealand 31(4):845-864. https://doi.org/10.1080/03014223.2001.9517680.

Mazzonia, E., and M. Vázquez. 2009. Desertification in Patagonia. Developments in Earth Surface Processes 13:351-377. https://doi.org/10.1016/S0928-2025(08)10017-7.

Mazzoni, E., and J. Rabassa. 2013. Types and internal hydro-geomorphologic variability of mallines (wet-meadows) of Patagonia: Emphasis on volcanic plateaus. Journal of South American Earth Sciences 46:170-182. https://doi.org/ 10.1016/j.jsames.2011.08.004.

Monjeau, J. A., E. C. Birney, L. Ghermandi, R. S. Sikes, L. Margutti, and C. J. Phillips. 1998. Plants, small mammals, and the hierarchical landscape classifications of Patagonia. Landscape Ecology 13(5):285-306. https://doi.org/10.1023/A: 1008012613305.

Morriss, S., C. Massey, R. Flett, F. Alpass, and F. Sligo. 2006. Mediating technological learning in agricultural innovation 
systems. Agricultural Systems 89(1):26-46. https://doi.org/10.1016/j.agsy.2005.08.002.

Movia, C. P., G. H. Ower, and C. E. Pérez. 1982. Estudio de la vegetación natural de la provincia del Neuquén, Tomo I: Relevamiento. Subsecretaría de Recursos Naturales, Ministerio de Economía y Hacienda, Provincia del Neuquén. Pp. 164.

Norgaard, R. B. 1981. Sociosystem and Ecosystem Coevolution in the Amazon. Journal of Environmental Economics and Management 8:238-254. https://doi.org/10.1016/0095-0696(81)90039-5.

Nosetto, M. D., E. G. Jobbágy, and J. M. Paruelo. 2006. Carbon sequestration in semi-arid rangelands: comparison of Pinus ponderosa plantations and grazing exclusion in NW Patagonia. Journal of Arid Environments 67(1):142-156. https://doi.org/10.1016/j.jaridenv.2005.12.008.

Oliva, G., D. Bran, J. Gaitán, D. Ferrante, V. Massara, G. G. Martínez, E. Adema, M. Enrique, E. Domínguez, and P. Paredes. 2019. Monitoring drylands: The MARAS system. Journal of Arid Environments 161:55-63. https://doi.org/ 10.1016/j.jaridenv.2018.10.004.

Oñatibia, G. R., M. R. Aguiar, and M. Semmartin. 2015. Are there any trade-offs between forage provision and the ecosystem service of $C$ and $N$ storage in arid rangelands? Ecological Engineering 77:26-32. https://doi.org/10.1016/ j.ecoleng.2015.01.009.

Oñatibia, G. R., and M. R. Aguiar. 2016. Continuous moderate grazing management promotes biomass production in Patagonian arid rangelands. Journal of Arid Environments 125:73-79. https://doi.org/10.1016/j.jaridenv.2015.10.005.

Oyarzabal, M., J. R. Clavijo, L. J. Oakley, F. Biganzoli, P. M. Tognetti, I. M. Barberis, H. M. Maturo, M. R. Aragón, P. I. Campanello, D. E. Prado, M. Oesterheld, and R. J. C. León. 2018. Unidades de vegetación de la Argentina. Ecología Austral 28:40-63. https://doi.org/10.25260/EA.18.28.1.0.399.

Paruelo, J. M., E. G. Jobbágy, and O. E. Sala. 1998. Biozones of Patagonia (Argentina). Ecología Austral 8:145-153.

Pastur, G. M., P. L. Peri, M. V. Lencinas, M. García-Llorente, and B. Martín-López. 2016. Spatial patterns of cultural ecosystem services provision in Southern Patagonia. Landscape Ecology 31(2):383-399. https://doi.org/10.1007/s10980015-0254-9.

Pedrana, J., J. Bustamante, A. Travaini, and A. Rodríguez. 2010. Factors influencing guanaco distribution in southern Argentine Patagonia and implications for its sustainable use. Biodiversity and Conservation 19(12):3499-3512. https: //doi.org/10.1007/s10531-010-9910-1.

Perelman, S. B., R. J. C. León, and J. P. Bussacca. 1997. Floristic changes related to grazing intensity in a Pantagonian shrub steppe. Ecography 20:400-406. https://doi.org/10.1111/j.1600-0587.1997.tb00385.x.

Pérez León, N., O. Bruzzone, and M. H. Easdale. 2020. A framework to tackling the synchrony between social and ecological phases of the annual cyclic movement of transhumant pastoralism. Sustainability 12(8):3462. https: //doi.org/10.3390/su12083462.

Peri, P. L., Y. M. Rosas, B. Ladd, S. Toledo, R. G. Lasagno, and G. Martínez Pastur. 2018. Modelling soil carbon content in South Patagonia and evaluating changes according to climate, vegetation, desertification and grazing. Sustainability 10(2):438. https://doi.org/10.3390/su10020438.

Raffestin, C. 1980. Pour une géographie du pouvoir. LITEC, Paris.

Raymond, C. M., I. Fazey, M. S. Reed, L. C. Stringer, G. M. Robinson, and A. C. Evely. 2010. Integrating local and scientific knowledge for environmental management. Journal of Environmental Management 91(8):1766-1777. https: //doi.org/10.1016/j.jenvman.2010.03.023.

Reynolds, J. F., and D. M. Stafford Smith. 2002. Global desertification: Do humans cause deserts?, Dahlem University Press, Berlin. Pp. 430.

Reynolds, J. F., D. M. S. Smith, E. F. Lambin, B. L. Turner, M. Mortimore, S. P. Batterbury, T. E. Downing, H. Dowlatabadi, R. J. Fernández, J. E. Herrick, and E. Huber-Sannwald. 2007. Global desertification: building a science for dryland development. Science 316(5826):847-851. https://doi.org/10.1126/science.1131634.

Reynolds, J. F., A. Grainger, D. M. Stafford Smith, G. Bastin, L. García-Barrios, R. J. Fernández, M. A. Janssen, N. Jürgens, R. J. Scholes, A. Veldkamp, and M. M. Verstraete. 2011. Scientific concepts for an integrated analysis of desertification. Land Degradation and Development 22(2):166-183. https://doi.org/10.1002/ldr.1104.

Roig, F. A., J. Anchorena, O. Dollenz, A. M. Faggi, and E. Méndez. 1985. Las comunidades vegetales de la Transecta Botánica de la Patagonia Austral. Primera parte. Pp. 350-456 en O. Boelcke, D. M. Moore and F. A. Roig (eds.). La vegetación del área continental. Transecta Botánica de la Patagonia Austral. Consejo Nacional de Investigaciones Científicas y Técnicas, Instituto de La Patagonia y The Royal Society. Buenos Aires.

Rozzi, R. 2013. Biocultural ethics: from biocultural homogenization toward biocultural conservation. Pp. 9-32 en Linking ecology and ethics for a changing World. Springer, Dordrecht. https://doi.org/10.1007/978-94-007-7470-4_2.

Safriel, U., and Z. Adeel. 2005. Dryland systems (chapter 22). In R. Hassan, R. Scholes and N. Ash (eds.). Millennium Ecosystem Assessment. Ecosystems and Human Well-Being: Current State and Trends. Volume 1. World Resources Institute, Island Press, Washington, DC.

Schorr, A. G., and M. F. Segui. 2008. Zonas Agroeconómicas Homogéneas de Patagonia Sur: Chubut, Santa Cruz y Tierra del Fuego. Serie Estudios Socioeconómicos de la Sustentabilidad de los Sistemas de Producción y Recursos Naturales, INTA. Pp. 97.

Soriano, A. 1956. Los distritos florísticos de la Provincia Patagónica. Revista de Investigaciones Agrícolas 10:323-347.

Soriano, A. 1983. Deserts and semi-deserts of Patagonia. Pp. 423-459 in N. E. West (ed.). Temperate Deserts and Semideserts. Elsevier Scientific Publishing Company, Amsterdam

Soriano, A., and J. M. Paruelo. 1992. Biozones: vegetation units defined by functional characters identifiable with the 
aid of satellite sensor images. Global Ecology and Biogeography Letters 2:82-89. https://doi.org/10.2307/2997510.

Soulé, M. E. 1985. What is conservation biology? BioScience 35(11):727-734. https://doi.org/10.2307/1310054.

Stafford Smith, D. M. 2008. The 'desert syndrome' - causally-linked factors that characterise outback Australia. The Rangeland Journal 30:3-14. https://doi.org/10.1071/RJ07063.

Stoddart, L. A., A. D. Smith, and T. W. Box. 1975. Range management, 3rd ed. New York: McGraw-Hill Book Company.

Thomas, D. S. 1997. Science and the desertification debate. Journal of Arid Environments 37(4):599-608. https://doi.org/ 10.1006/jare.1997.0293.

Torres, L., E. M. Abraham, C. Rubio, C. Barbero-Sierra, and M. Ruiz-Pérez. 2015. Desertification research in Argentina. Land Degradation and Development 26(5):433-440. https://doi.org/10.1002/ldr.2392.

United Nations Educational, Scientific and Cultural Organization (UNESCO). 2019. Transhumance, the seasonal droving of livestock along migratory routes in the Mediterranean and in the Alps. URL: tinyurl.com/yb8bap38 (útimo acceso: 15 de mayo de 2021). https://doi.org/10.18356/1db7b282-es.

Vallejos, M., S. Aguiar, G. Baldi, M. E. Mastrángelo, F. Gallego, M. Pacheco-Romero, D. Alcaraz-Segura, and J. M. Paruelo. 2019. Social-Ecological Functional Types: connecting people and ecosystems in the Argentine Chaco. Ecosystems 23 : 471-484. https://doi.org/10.1007/s10021-019-00415-4.

Van Dyke, F. 2008. Conservation biology: foundations, concepts, applications. New York, Springer Science and Business Media. Pp. 478.

Verón, S. R., J. M. Paruelo, and M. Oesterheld. 2006. Assessing desertification. Journal of Arid Environments 66(4): 751-763. https://doi.org/10.1016/j.jaridenv.2006.01.021.

Verón, S. R., and J. M. Paruelo. 2010. Desertification alters the response of vegetation to changes in precipitation. Journal of Applied Ecology 47(6):1233-1241. https://doi.org/10.1111/j.1365-2664.2010.01883.x.

Villagra, E. S., M. H. Easdale, C. G. Giraudo, and G. L. Bonvissuto. 2015. Productive and income contributions of sheep, goat, and cattle, and different diversification schemes in smallholder production systems of Northern Patagonia, Argentina. Tropical Animal Health and Production 47(7): 1373-1380. https://doi.org/10.1007/s11250-015-0873-9.

Vogt, J. V., U. Safriel, G. Von Maltitz, Y. Sokona, R. Zougmore, G. Bastin, and J. Hill. 2011. Monitoring and assessment of land degradation and desertification: towards new conceptual and integrated approaches. Land Degradation and Development 22(2):150-165. https://doi.org/10.1002/ldr.1075.

Walker, B. H., and D. Salt. 2006. Resilience thinking. Sustaining Ecosystems and People in a Changing World. Island Press, Washington, USA. 